\title{
Mesenchymal Stem Cells for the Treatment of Liver Disease: Present and Perspectives
}

\author{
Seong Hee Kang ${ }^{1,2,3}$, Moon Young Kim ${ }^{1,2}$, Young Woo Eom ${ }^{1,2}$, and Soon Koo Baik ${ }^{1,2,3}$ \\ ${ }^{1}$ Department of Internal Medicine, ${ }^{2}$ Cell Therapy and Tissue Engineering Center, and ${ }^{3}$ Institute of Evidence Based Medicine, Yonsei University \\ Wonju College of Medicine, Wonju, Korea
}

\begin{abstract}
Mesenchymal stem cell transplantation is an emerging therapy for treating chronic liver diseases. The potential of this treatment has been evaluated in preclinical and clinical studies. Although the mechanisms of mesenchymal stem cell transplantation are still not completely understood, accumulating evidence has revealed that their immunomodulation, differentiation, and antifibrotic properties play a crucial role in liver regeneration. The safety and therapeutic effects of mesenchymal stem cells in patients with chronic liver disease have been observed in many clinical studies. However, only modest improvements have been seen, partly because of the limited feasibility of transplanted cells at present. Here, we discuss several strategies targeted at improving viable cell engraftment and the potential challenges in the use of extracellular vesicle-based therapies for liver disease in the future. (Gut Liver 2020;14:306-315)
\end{abstract}

Key Words: Mesenchymal stromal cells; Liver disease; Cell transplantation; Cell survival

\section{INTRODUCTION}

Chronic liver injury, such as that arising from viral infection, alcohol abuse, or metabolic diseases, causes liver cirrhosis and failure. ${ }^{1,2}$ The ultimate treatment for end-stage cirrhosis is liver transplantation. ${ }^{3}$ However, transplants are not readily available in many countries, and in countries where transplants are available, organ shortages and high costs related with transplantation make this an impractical option for many patients.

Stem cell transplantation has been proposed as a potential strategy for patients with hepatic diseases to prevent progression and treat those with advanced fibrosis. Stem cell transplantation including hematopoietic, induced pluripotent, and mesenchymal stem cells (MSCs) can be manipulated for division into hepatocyte-like cells both in vitro and in vivo. ${ }^{4,5}$ Of these cell types, MSCs have been shown to have the advantages of being obtained relatively easily and possessing low immunogenicity. ${ }^{6}$ They have self-renewal ability and can differentiate into cells of various lineages, including osteoblasts, adipocytes, and chondrocytes. ${ }^{7}$ Additionally, MSCs are safe in terms of ethical concerns because they do not originate from somatic cells. Furthermore, MSC transplantation has been considered safe and widely assessed in clinical settings of various diseases with promising results. $^{8}$

The purpose of this review is to present the therapeutic effects of MSCs in liver diseases to address questions regarding efficacy, safety, and possible risks involved, as well as to discuss recent clinical advances involving clinical MSC-based therapies, opening a new path toward further studies.

\section{OVERVIEW OF MSC TRANSPLANTATION}

\section{Definition and sources of MSC transplantation}

MSCs can differentiate into either mesodermal or ectodermal cells, resulting in adherent multipotent fibroblast-type stem cells. ${ }^{9}$ Different investigators define MSC characteristics in varying ways. To address this problem, the International Society for Cellular Therapy recommends a set of three criteria to define human MSCs; $;^{10}$ adherence to plastic, specific surface antigen expression, and multipotent differentiation potential (Table 1). MSCs can be isolated from most organs or tissues, including bone marrow (BM), umbilical cord blood (UCB), adipose tissue (AT), peripheral blood, trabecular bone, synovial membrane, cartilage, and muscle. ${ }^{11,12}$ Among these, three main sources have been demonstrated as capable of treating liver disease: BM-MSCs, UCB-MSCs, and AT-MSCs. Generally, MSCs derived

Correspondence to: Soon Koo Baik

Department of Internal Medicine, Yonsei University Wonju College of Medicine, 20 Ilsan-ro, Wonju 26426, Korea

Tel: +82-33-741-1223, Fax: +82-33-741-0951, E-mail: baiksk@yonsei.ac.kr

Received on September 15, 2018. Revised on December 14, 2018. Accepted on December 23, 2018. Published online October 8, 2019. pISSN 1976-2283 eISSN 2005-1212 https://doi.org/10.5009/gnl18412

(c) This is an Open Access article distributed under the terms of the Creative Commons Attribution Non-Commercial License (http://creativecommons.org/licenses/by-nc/4.0) which permits unrestricted non-commercial use, distribution, and reproduction in any medium, provided the original work is properly cited. 
Table 1. Criteria of the International Society for Cellular Therapy for Defining MSCs

\begin{tabular}{|c|c|c|c|}
\hline \multirow{2}{*}{ Adherence to plastic } & \multicolumn{2}{|c|}{ Surface antigen expression } & \multirow{2}{*}{ Multipotent differentiation } \\
\hline & Positive ( $\geq 95 \%$ ) & Negative $(\leq 2 \%)$ & \\
\hline \multirow{4}{*}{$\begin{array}{l}\text { Adherence to plastic in standard } \\
\text { culture conditions }\end{array}$} & CD73 & CD14/CD11b & \multirow{4}{*}{$\begin{array}{l}\text { Differentiation potential into osteoblasts, } \\
\text { adipocytes, chondroblasts, which is } \\
\text { demonstrated by staining of in vitro } \\
\text { cultured cells }\end{array}$} \\
\hline & CD90 & $\mathrm{CD} 79 \alpha / \mathrm{CD} 19$ & \\
\hline & CD105 & CD34 & \\
\hline & & HLA-DR & \\
\hline
\end{tabular}

MSCs, mesenchymal stem cells; HLA-DR, human leukocyte antigen-DR isotype.

from these three sources are well-known to express similar surface antigens, whereas their morphology and proliferation rate vary. ${ }^{13}$ First, although BM is the largest source, BM-derived MSCs may have restricted clinical use because of the invasive procedure required for their isolation, insufficient cell number, and reduced differentiation ability with increasing age. ${ }^{14}$ Next, UCB-MSCs, which can be obtained using less-invasive methods, have been addressed as a substitute source. ${ }^{7}$ UCB-MSCs are easy to obtain for collection after delivery; further, they remain viable even after cryopreservation. Finally, AT-MSCs have several advantages. They have the highest proliferative capacity and carry the benefits of requiring a less-invasive procedure and are easily obtained through simple lipo-aspiration. ${ }^{15}$ Until now, BM has been the most common source in clinical settings. However, important concerns regarding the choice of MSC source must still be addressed to make stem cell therapies applicable for liver disease.

\section{Therapeutic mechanisms of MSC transplantation in liver disease}

\section{1) Trans-differentiation into hepatocyte-like cells}

Hepatocyte-like cells derived from MSCs have been considered substitute sources for liver regeneration. ${ }^{16}$ Hepatic differentiation of MSCs is influenced by several factors. First, MSCs can be differentiated into hepatocyte-like cells by processing with many cytokines and growth factors such as hepatocyte growth factor (HGF), fibroblast growth factor-2/4, epidermal growth factor, oncostatin $\mathrm{M}$, leukemia inhibitory factor, dexamethasone, insulin-transferrin-selenium, or nicotinamide. ${ }^{17}$ Injured liver tissue surrounded with the extracellular matrix (ECM) has been used as the location for MSC engraftment and differentiation because it has been shown that liver ECM triggers MSC differentiation. Additionally, co-culture with liver cells ${ }^{18}$ and pellet culture $^{19}$ can induce MSC differentiation into hepatocyte-like cells. However, the trans-differentiation of MSCs into hepatocytes occurs in less than $1 \%$ of the total liver mass in preclinical settings. ${ }^{20}$ A more efficient hepatocyte differentiation technique should be developed to utilize hepatocyte-differentiated MSCs for treating liver disease.

\section{2) Immunomodulation}

Chronic liver injury caused by inflammation is accompanied by infiltration of T cells, B cells, and monocytes (Fig. 1). ${ }^{21}$ It has been reported that immunosuppressive agents can be beneficial to liver regeneration before and after liver transplantation. ${ }^{22,23}$ In this respect, MSCs' immunomodulatory properties can have favorable effects in liver disease. First, MSCs can downregulate $\mathrm{T}$ cells by releasing various soluble factors, such as nitric oxide, prostaglandin E (PGE)-2, indoleamine 2, 3-dioxygenase, interleukin (IL)-6, IL-10, and human leukocyte antigen G. These factors can control the proliferation and functions of various immune cells and upregulate $\mathrm{T}_{\text {reg }}$ cells. ${ }^{24}$ MSCs can also inhibit the proliferation of $\mathrm{T}$ cells by directly interacting with T-lymphocytes. The immunosuppressive ability of MSCs is generated by a combination of cytokines such as interferon- $\gamma, \mathrm{IL}-1 \alpha$, and tumor necrosis factor (TNF)- $\alpha .^{25}$ These cytokines help some chemokines and immune cells stay in contact with the MSCs and regulate immune reactions. Additionally, MSCs can inhibit the activation of B cells, reducing levels of immunoglobulin. Co-culture with MSCs has been associated with a significant reduction of surface expression of chemokine receptor (CXCR4, CXCR7, and CXCR5). ${ }^{26}$ In addition, natural killer (NK) cells have been well-known as an important factor in immune reactions against viral infections and cancer. ${ }^{27}$ MSCs induce IL-2 expression, resulting in reduced IL-15 secretion from IL-2-induced NK cells by either cell-to-cell interactions or secretion of soluble factors such as PGE2 and transforming growth factor (TGF)- $\beta .^{28}$ Finally, MSCs have been shown to induce the polarization of inflammatory macrophages toward alternative macrophages. This alteration releases the soluble factors (i.e., IL-10 and IL1Ra) that improve liver injury. ${ }^{29}$

\section{3) Anti-fibrotic activities}

Chronic liver injury causes the trans-differentiation of quiescent hepatic stellate cells (HSCs) into fibrogenic myofibroblasts, producing excess ECM proteins and resulting in fibrosis. This proliferation of activated HSCs and collagen deposition can be attenuated with MSC treatment by indirect or direct cell-cell contact. In the indirect contact mechanism, several soluble factors (i.e., TGF- $\beta 3$, TNF- $\alpha$, IL-10, and HGF) secreted by MSCs 


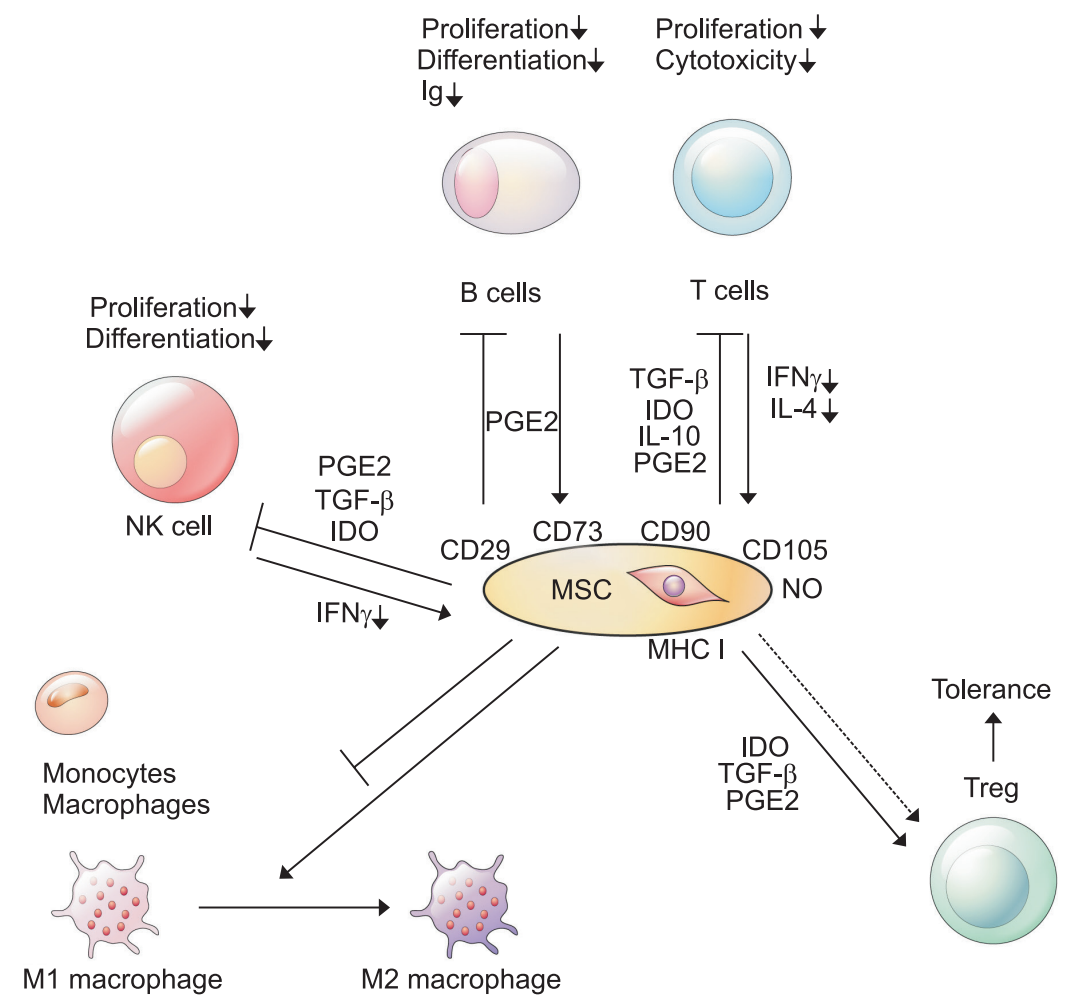

Fig. 1. Immunomodulation by mesenchymal stem cell (MSC) transplantation in liver disease occurs at multiple levels.

Ig, immunoglobulin; NK, natural killer; PGE, prostaglandin E; TGF, transforming growth factor; ID0, indoleamine 2,3-dioxygenase; IFN, interferon; IL, interleukin; MHC, major histocompatibility complex, NO, nitric oxide.

attenuate collagen synthesis, ${ }^{30,31}$ whereas HGF and nerve growth factor induce the apoptosis of HSCs. ${ }^{32}$ Next, MSCs co-cultured with HSCs inhibit the proliferation of HSCs and expression of $\alpha$-smooth muscle actin (SMA) through cell-to-cell contact. ${ }^{33}$ It is well-known that matrix metalloproteinases (MMPs) and tissue inhibitors of MMPs (TIMPs) contribute to both the progression and regression of liver fibrosis. In several fibrosis models, MSCs regulate the expression of MMPs (i.e., MMP-2, -9, -13, and -14) and TIMP-1; increasing the expression of MMPs and decreasing the expression of TIMPs. ${ }^{32,34}$

\section{ARE WE READY FOR MSC TRANSPLANTATION IN ROU- TINE CLINICAL PRACTICE?}

\section{Present efficacy and safety of MSC transplantation}

Several clinical trials have elucidated the advantages of MSC treatment in chronic liver disease (Table 2). ${ }^{35-52}$

A pilot study conducted by Mohamadnejad et al. ${ }^{35}$ in 2007 showed that infusion of autologous MSCs was safe and feasible for treatment in four patients with decompensated liver cirrhosis. Model for End-Stage Liver Disease (MELD) scores improved in three out of four patients at 6 months and two of them remained stable up to 12 months. In a phase 2 study conducted by Amer et al. ${ }^{37}$ published in 2011, 40 patients with hepatitis Crelated liver cirrhosis were randomized into two groups of 20 patients: the first group received autologous BM-derived MSCs, while the second group received the best supportive treatment.
Compared to the second group, the first group showed significant improvement in Child-Pugh and MELD scores, which was maintained for 6 months. Shi et al ${ }^{40}$ reported that 24 patients with hepatitis B-related acute-on-chronic liver failure were treated with intravenous UCB-MSC transfusions, and 19 patients were treated with saline as controls. The UCB-MSC transfusions significantly increased the survival rates for 72 weeks and no significant side effects were observed until the end of the follow-up. Similar results were obtained by Amin et al. ${ }^{42}$ who showed that intrasplenic autologous transplantation improved liver function in 20 patients with hepatitis C-related liver cirrhosis, as determined by significant decreases in the total bilirubin, aspartate transaminase, and alanine aminotransferase levels, and prothrombin time as well as a significant increase in the albumin at 6-month follow-up. An open-labe trial published by Jang et al. ${ }^{45}$ in 2014 showed beneficial effects of autologous BM-MSC transplantation via the hepatic artery for treating alcohol abuse-related liver cirrhosis. Histological improvements were observed in 54.5\% of patients; the ChildPugh score was improved from 7.1 to 5.4, and the levels of fibrosis-related markers including TGF- $\beta 1$, type 1 collagen, and $\alpha$-SMA were significantly decreased 12 weeks after the second injection. Recently, a randomized phase 2 trial reported that hepatic arterial injections of autologous BM-MSCs for 72 patients with alcohol-related liver cirrhosis could alleviate liver fibrosis and improve Child-Pugh scores. ${ }^{49}$ A recent clinical study also reported the feasibility, safety, and tolerability of MSC therapy 


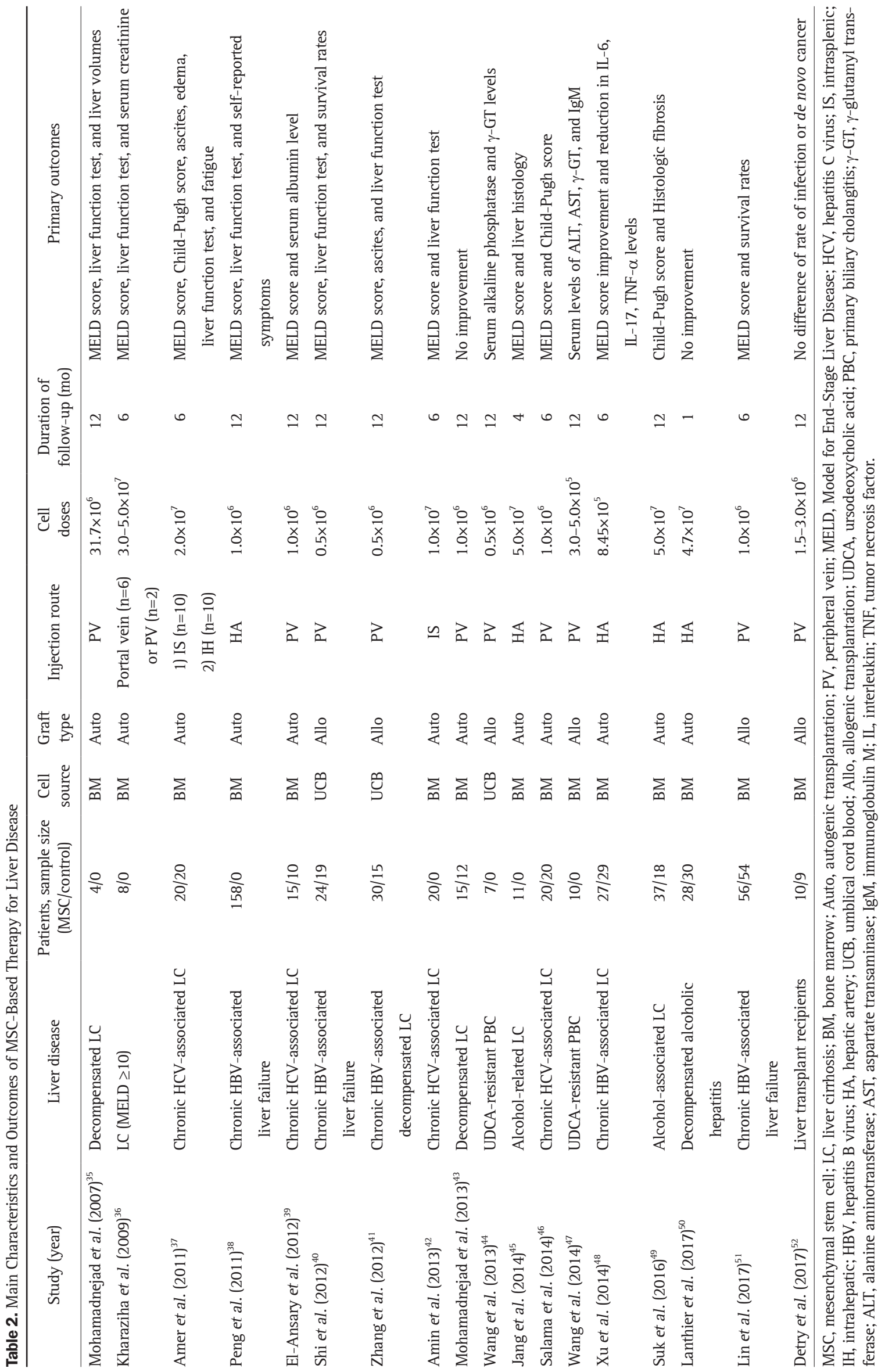


in liver transplant recipients $(\mathrm{n}=10)$. Treatment with MSCs has been proposed to have a potential beneficial effect on ischemia/ reperfusion injury. This study opened a path for utilizing MSCs as a potential future therapy for liver transplant recipients who require life-long immunosuppression. ${ }^{52}$

However, negative results were obtained in two clinical studies. Mohamadnejad et al. ${ }^{43}$ reported that autologous BM-MSC transplantation $(n=15)$ injected through the peripheral vein likely has no beneficial effect compared to controls $(n=12)$. The absolute changes in Child-Pugh score, MELD scores, serum transaminase levels, and liver volumes did not differ between the MSC-treated and control groups at 12 months of follow-up. Another study reported that 28 patients with alcoholic hepatitis were treated with autologous BM-derived CD $34^{+}$stem cells and MSCs and 30 patients were treated with supportive therapy only. No significant difference between the two groups was observed in terms of the proliferative hepatocyte number in liver biopsy at the 4-week follow-up. However, patients who received stem cell treatment showed more active liver macrophagic expansion as compared to those who received standard treatment. $^{50}$

Most studies have suggested that stem cell therapy is safe and effective in patients with liver disease. However, the size and nature of the trial design of many of these clinical studies meant that meaningful conclusions could not be drawn, and thus, their efficacy has yet to be confirmed.

\section{Routes of MSC transplantation}

There are conflicting data about engraftment of transplanted MSCs and some concerns regarding their fibrogenic potential have been raised. It seems that these unwanted effects depend on the route and dose of MSCs infusion. ${ }^{53,54}$ Though the effectiveness is reported to vary slightly depending on the injection route, MSCs can be transplanted into the liver through intravenous, intrahepatic, intraperitoneal, intrasplenic, or portal vein injection. The peripheral vein has been known as the most common transplantation route, followed by the hepatic artery, intrasplenic injection, intrahepatic injection, and portal vein. BM-MSCs administered through the peripheral vein have been shown to migrate well into liver parenchyma in the context of chronic injury in vivo. In contrast, limited MSC engraftment has been observed in an acute injury environment. ${ }^{54}$ In addition, MSCs endured in liver tissues when injected through the intrahepatic artery, demonstrating that MSCs were present and did not differentiate into hepatocytes. Additionally, intraportal infusion was more efficient than the peripheral route in clinical trials. ${ }^{37}$ However, direct approaches, such as via the portal vein or hepatic artery, may carry the risk of portal hypertensive bleeding following cell injection. ${ }^{55}$ Overall, evidence provided by most of these clinical studies has been quite lacking until now.

\section{Potential risks of MSC transplantation}

So far, clinical and preclinical studies about MSC treatment for chronic liver disease have been conducted, and several problems must be cautiously considered, including the possibility of carcinogenesis and viral transmission. MSCs can secrete various growth factors that encourage tumor cell growth and angiogenesis. $^{56}$ Previous experimental studies showed that the tendency for malignant formation depended on the number of passages. For example, in mouse MSCs, chromosomal abnormalities and transformation into malignant cells, such as sarcoma, have been observed after more than three passages. ${ }^{57,58}$ Furthermore, MSCs may demonstrate telomeric deletions upon numerous passages. ${ }^{59}$ Although the malignant transformation of human MSCs has not yet been observed in clinical trials, the follow-up period was too short for the occurrence of a tumor to be evident in most of them. Therefore, chromosomal integrity must be analyzed before MSCs transplantation to ensure the safety of the procedure.

In contrast to autotransplantation, allotansplantation may involve the risk of viral transmission to the patients. ${ }^{41}$ Although viral transmission of parvovirus B19 into BM cells was demonstrated in vitro, B19-positive MSC-related viremia has not yet been reported in humans. However, no information is yet available on the transmission of herpes simplex virus (HSV) and cytomegalovirus (CMV) via MSCs in vivo. Therefore, both MSC recipients and donors may need to be screened for parvovirus B19, HSV, and CMV because of the possibility of infection in immunosuppressed patients.

\section{FUTURE DIRECTIONS OF MSC TRANSPLANTATION IN LIVER DISEASE}

Clinical studies have demonstrated only a moderate benefit, at least in part because of the limited viability of the transplanted cells, irrespective of the cell source. Even some reports reported that less than $1 \%$ of transplanted cells may survive because of the inflexibilities of the microenvironment they encounter upon transplantation..$^{60}$ In this section, we will review the strategies that have been utilized to improve the effects of cell therapy in MSC transplantation (Fig. 2).

\section{Tissue engineering}

The tissue engineering approach aims to allow cell homing and adaptation in the transplanted organ before starting their regeneration, resulting in improved cell survival. The ECM plays a crucial role in cellular organization and function. ${ }^{61}$ Several approaches have been investigated, including co-culture and the development of 3-dimensional (3D) systems. ${ }^{62,63}$ It is possible that cells grown in 3D systems would behave more like cells in vivo and be implanted directly. These 3D systems can be classified into scaffold-based and scaffold-free systems.

Several synthetic polymers, including poly lactic co-glycolic 


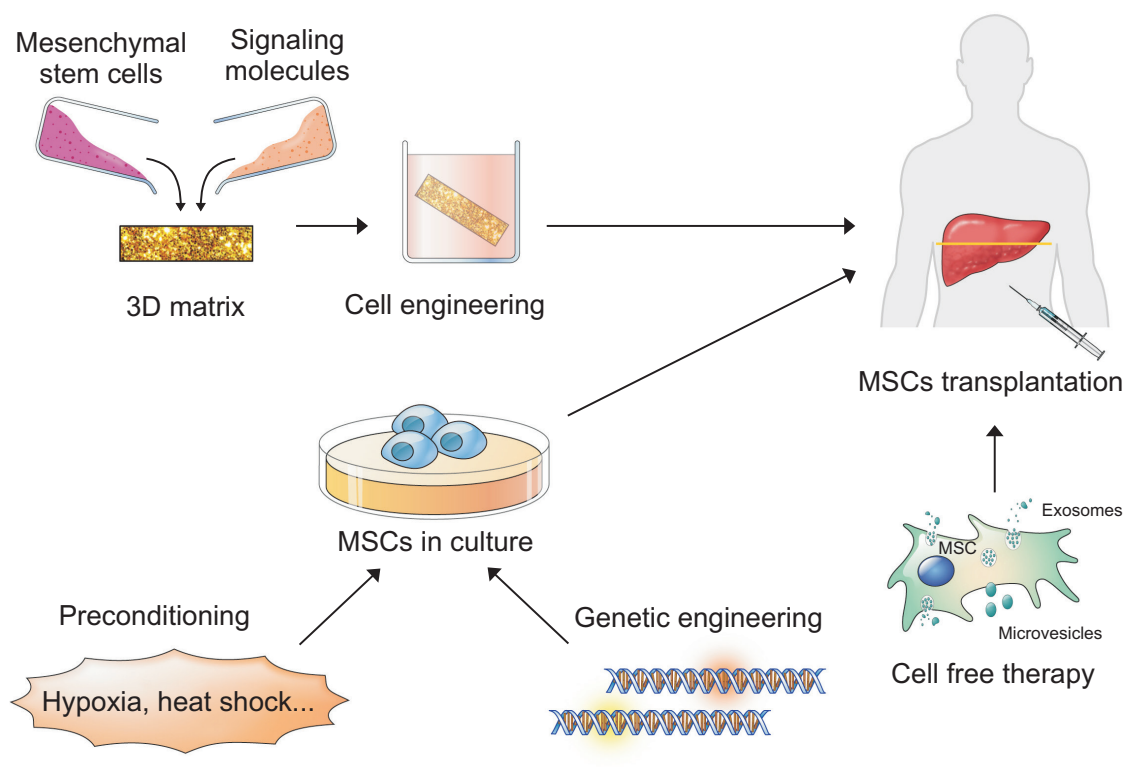

Fig. 2. Strategies for improving the efficacy of mesenchymal stem cell (MSC) therapies.

3D, 3-dimensional. acid (PLGA), and natural materials such as collagen, have been assessed for their ability to increase the expression of hepatocyte-specific genes in MSCs during hepatic differentiation. ${ }^{64}$ Indeed, modulation of liver function was shown in co-culture of BM-derived MSCs and isolated fresh hepatocytes on a PLGA scaffold. The greatest effect was observed in performance using a 1:5 ratio of MSCs to hepatocytes in vitro and in vivo. ${ }^{65} \mathrm{In}$ addition to scaffold-based 3D systems, the roles of biological scaffolds, such as decellularized tissue, have been evaluated by several groups. Decellularized liver tissue forms an ECM scaffold that can improve MSC engraftment by providing a more physiological environment. ${ }^{66}$

\section{Preconditioning to improve cell resistance}

During treatment, MSCs are transplanted into pathological disease conditions. In other words, pathological conditions put implanted cells in severe acidic, oxidative, and nutritional stresses. ${ }^{67}$ In this regard, modifying donor cells before transplant helps those cells resist harsh conditions, resulting in improved cell function. Several strategies for preconditioning include promoting a broad pro-survival response through exposing cells to a physical or environmental shock and pharmacological modulators of targeted molecules. 68,69

First, thermal preconditioning at $42^{\circ} \mathrm{C}$ for 1 to 2 hours before transplantation has been demonstrated to promote cell survival in vivo. This effect is related to the induction of heat shock protein expression, which inhibits apoptotic pathways. ${ }^{70,71}$ Next, hypoxia, an important feature of MSCs, has been shown to play a crucial role in maintaining stem cell fate, self-renewal, and multi-potency, and cultivating MSCs under hypoxia is an important preconditioning step because it mimics the natural microenvironment of $\mathrm{BM}^{72}$ In this respect, hypoxic precon- ditioning strategies have been developed to promote defense mechanisms against oxidative stress. Priming MSCs in $0.5 \%$ to 3\% low oxygen may help to increase engraftment success by inhibiting apoptotic pathways including Akt, B-cell lymphoma (Bcl)-2, and hypoxia-inducible factor (HIF)-1 $\alpha$ and the upregulation of chemokine receptors (i.e., CXCR4 and CX3CR1). ${ }^{72-74}$ Although some data have been accumulated by preclinical studies, the response of MSCs to hypoxic conditions is rather contradictory, indicating both damaging and ameliorating effects. Pharmacologic preconditioning of cells before transplantation is another emerging strategy to maintain cell viability after transplantation. For example, antioxidants and HIF- $1 \alpha$ stabilizers contribute to cell survival, while antimycin and mitochondrial electron transport inhibitors have also been described to promote cell survival by activating mitochondrial death pathways. ${ }^{75}$

\section{Genetic engineering}

Several approaches have been investigated to promote the expression of proteins involved in homing of donor cells. ${ }^{76}$ MSCs have been shown to express low levels of molecules including the homing factor stromal cell-derived factor-1 and chemokine receptors (i.e., CXCR4 and CCR 1 receptors). ${ }^{77}$ Genetic manipulation of pro-survival or anti-apoptotic genes including Bcl-2, protein kinase B (Akt/PBK), HGF, and survivin increased MSC survival in vivo. ${ }^{78-81}$ It is also known that miRNA can regulate mRNAs, modulating the cellular gene networks, including those involved in cell survival. miRNA overexpression has been shown to enhance MSC survival. ${ }^{82,83}$ However, several problems, including the risk of carcinogenesis, must be carefully considered when applying genetic manipulations. 


\section{Extracellular vesicles as cell-free therapy}

MSCs can secrete soluble molecules with a paracrine effect or release more complex structures called extracellular vesicles (EVs). ${ }^{84}$ EVs exert many of their effects by interaction with the cell surface, internalization, or fusion with the cell membrane. These EVs can be engineered to improve the expression of anticipated activities or introduce specific effector molecules. ${ }^{85,86}$

MSC-derived EVs improved hepatic injury and inflammation by inactivating the TGF- $\beta /$ Smad signaling pathway in a $\mathrm{CCl}_{4}{ }^{-}$ induced fibrosis model. ${ }^{87}$ Moreover, EVs derived from human MSCs preserve at least some of the immunomodulatory properties of the cells. A recent study also showed that MSC-derived induced pluripotent stem cell (iPSC)-EVs retain the characteristics of EVs that are usually obtained from tissue-derived MSCs, regardless of origin. ${ }^{88}$ It has been reported that MSC-iPSC-EVs can directly fuse with hepatocytes, increasing the activity of sphingosine kinase-1 and sphingosine-1-phosphate levels and affecting hepatocyte proliferation. ${ }^{89}$ From this perspective, EVs could be a more encouraging therapeutic strategy because they characterize a physically different fraction and transport signals with more predictable effects. However, the complex functions of EVs are still largely unknown. Moreover, further studies are needed to determine how long circulating MSC-EVs survive after administration and what recognition pathways are used by target cells.

\section{CONCLUSIONS}

MSC regenerative therapy in chronic liver disease has been shown to be effective via their immunomodulation, differentiation, and anti-fibrosis properties. Many clinical studies have demonstrated the efficacy of MSCs in treating injured hepatocytes by ameliorating tissue fibrosis and improving liver function. However, several concerns remain, including the low migration, poor cell survival, and the risk of carcinogenesis and viral transmission. We reviewed several strategies to enhance their efficacy, including modifying the culture environment and/or priming MSCs along with genetic engineering of cells. In addition, EVs produced by MSCs seem to have therapeutic benefits as a cell-free cell therapy in MSC-based transplantation by preserving at least some of the immunomodulatory properties of the cells.

The prospects of MSC-based cell therapy for chronic liver disease will be determined by standardizing the cell source, culture conditions, administration route, and the outcomes of future large-scale clinical trials.

\section{CONFLICTS OF INTEREST}

No potential conflict of interest relevant to this article was reported.

\section{ACKNOWLEDGEMENTS}

This research was supported by the Basic Science Research Program through the National Research Foundation of Korea (2017R1A5A2015369, 2017R1A2B4009199, 2017R1D1A1A0 2019212, and 2018R1C1B5044890) and a grant of the Korea Health Technology R\&D Project through the Korea Health Industry Development Institute (KHIDI), funded by the Ministry of Health \& Welfare, Republic of Korea (HI15C2364 and HI17C1365).

\section{REFERENCES}

1. Shim KY, Eom YW, Kim MY, Kang SH, Baik SK. Role of the reninangiotensin system in hepatic fibrosis and portal hypertension. Korean J Intern Med 2018;33:453-461.

2. Kim G, Kim MY, Baik SK. Transient elastography versus hepatic venous pressure gradient for diagnosing portal hypertension: a systematic review and meta-analysis. Clin Mol Hepatol 2017;23:34-41.

3. Kang SH, Kim MY, Baik SK. Novelties in the pathophysiology and management of portal hypertension: new treatments on the horizon. Hepatol Int 2018;12:112-121.

4. Si-Tayeb K, Noto FK, Nagaoka M, et al. Highly efficient generation of human hepatocyte-like cells from induced pluripotent stem cells. Hepatology 2010;51:297-305.

5. Shu SN, Wei L, Wang JH, Zhan YT, Chen HS, Wang Y. Hepatic differentiation capability of rat bone marrow-derived mesenchymal stem cells and hematopoietic stem cells. World J Gastroenterol 2004;10:2818-2822.

6. Kim G, Eom YW, Baik SK, et al. Therapeutic effects of mesenchymal stem cells for patients with chronic liver diseases: systematic review and meta-analysis. J Korean Med Sci 2015;30:1405-1415.

7. Secunda R, Vennila R, Mohanashankar AM, Rajasundari M, Jeswanth S, Surendran R. Isolation, expansion and characterisation of mesenchymal stem cells from human bone marrow, adipose tissue, umbilical cord blood and matrix: a comparative study. Cytotechnology 2015;67:793-807.

8. Ji ST, Kim H, Yun J, Chung JS, Kwon SM. Promising therapeutic strategies for mesenchymal stem cell-based cardiovascular regeneration: from cell priming to tissue engineering. Stem Cells Int 2017;2017:3945403.

9. Heo JS, Choi SM, Kim HO, et al. Neural transdifferentiation of human bone marrow mesenchymal stem cells on hydrophobic polymer-modified surface and therapeutic effects in an animal model of ischemic stroke. Neuroscience 2013;238:305-318.

10. Dominici M, Le Blanc K, Mueller I, et al. Minimal criteria for defining multipotent mesenchymal stromal cells. The International Society for Cellular Therapy position statement. Cytotherapy 2006;8:315-317.

11. Zarrabi M, Mousavi SH, Abroun S, Sadeghi B. Potential uses for cord blood mesenchymal stem cells. Cell J 2014;15:274-281. 
12. Ribeiro A, Laranjeira P, Mendes S, et al. Mesenchymal stem cells from umbilical cord matrix, adipose tissue and bone marrow exhibit different capability to suppress peripheral blood B, natural killer and T cells. Stem Cell Res Ther 2013;4:125.

13. Kern S, Eichler H, Stoeve J, Klüter H, Bieback K. Comparative analysis of mesenchymal stem cells from bone marrow, umbilical cord blood, or adipose tissue. Stem Cells 2006;24:1294-1301.

14. Liu WH, Song FQ, Ren LN, et al. The multiple functional roles of mesenchymal stem cells in participating in treating liver diseases. J Cell Mol Med 2015;19:511-520.

15. Banas A, Yamamoto Y, Teratani T, Ochiya T. Stem cell plasticity: learning from hepatogenic differentiation strategies. Dev Dyn 2007;236:3228-3241.

16. Eom YW, Shim KY, Baik SK. Mesenchymal stem cell therapy for liver fibrosis. Korean J Intern Med 2015;30:580-589.

17. Schwartz RE, Reyes M, Koodie L, et al. Multipotent adult progenitor cells from bone marrow differentiate into functional hepatocyte-like cells. J Clin Invest 2002;109:1291-1302.

18. Lange C, Bassler P, Lioznov MV, et al. Liver-specific gene expression in mesenchymal stem cells is induced by liver cells. World J Gastroenterol 2005;11:4497-4504.

19. Ong SY, Dai H, Leong KW. Inducing hepatic differentiation of human mesenchymal stem cells in pellet culture. Biomaterials 2006;27:4087-4097.

20. Dai LJ, Li HY, Guan LX, Ritchie G, Zhou JX. The therapeutic potential of bone marrow-derived mesenchymal stem cells on hepatic cirrhosis. Stem Cell Res 2009;2:16-25.

21. Kisseleva T, Brenner DA. The phenotypic fate and functional role for bone marrow-derived stem cells in liver fibrosis. J Hepatol 2012;56:965-972.

22. Dmitrewski J, Hubscher SG, Mayer AD, Neuberger JM. Recurrence of primary biliary cirrhosis in the liver allograft: the effect of immunosuppression. J Hepatol 1996;24:253-257.

23. Manousou P, Arvaniti V, Tsochatzis E, et al. Primary biliary cirrhosis after liver transplantation: influence of immunosuppression and human leukocyte antigen locus disparity. Liver Transpl 2010;16:64-73.

24. Sharma RR, Pollock K, Hubel A, McKenna D. Mesenchymal stem or stromal cells: a review of clinical applications and manufacturing practices. Transfusion 2014;54:1418-1437.

25. Ren G, Zhang L, Zhao X, et al. Mesenchymal stem cell-mediated immunosuppression occurs via concerted action of chemokines and nitric oxide. Cell Stem Cell 2008;2:141-150.

26. Glennie S, Soeiro I, Dyson PJ, Lam EW, Dazzi F. Bone marrow mesenchymal stem cells induce division arrest anergy of activated T cells. Blood 2005;105:2821-2827.

27. Sotiropoulou PA, Perez SA, Gritzapis AD, Baxevanis CN, Papamichail M. Interactions between human mesenchymal stem cells and natural killer cells. Stem Cells 2006;24:74-85.

28. Klyushnenkova E, Mosca JD, Zernetkina V, et al. T cell responses to allogeneic human mesenchymal stem cells: immunogenicity, tolerance, and suppression. J Biomed Sci 2005;12:47-57.
29. Lee KC, Lin HC, Huang YH, Hung SC. Allo-transplantation of mesenchymal stem cells attenuates hepatic injury through IL1Ra dependent macrophage switch in a mouse model of liver disease. J Hepatol 2015;63:1405-1412.

30. Parekkadan B, van Poll D, Megeed Z, et al. Immunomodulation of activated hepatic stellate cells by mesenchymal stem cells. Biochem Biophys Res Commun 2007;363:247-252.

31. Wang J, Bian C, Liao L, et al. Inhibition of hepatic stellate cells proliferation by mesenchymal stem cells and the possible mechanisms. Hepatol Res 2009;39:1219-1228.

32. Rabani V, Shahsavani M, Gharavi M, Piryaei A, Azhdari Z, Baharvand $\mathrm{H}$. Mesenchymal stem cell infusion therapy in a carbon tetrachloride-induced liver fibrosis model affects matrix metalloproteinase expression. Cell Biol Int 2010;34:601-605.

33. Chen S, Xu L, Lin N, Pan W, Hu K, Xu R. Activation of Notch1 signaling by marrow-derived mesenchymal stem cells through cell-cell contact inhibits proliferation of hepatic stellate cells. Life Sci 2011;89:975-981.

34. Semedo P, Correa-Costa M, Antonio Cenedeze M, et al. Mesenchymal stem cells attenuate renal fibrosis through immune modulation and remodeling properties in a rat remnant kidney model. Stem Cells 2009;27:3063-3073.

35. Mohamadnejad M, Alimoghaddam K, Mohyeddin-Bonab M, et al. Phase 1 trial of autologous bone marrow mesenchymal stem cell transplantation in patients with decompensated liver cirrhosis. Arch Iran Med 2007;10:459-466.

36. Kharaziha P, Hellström PM, Noorinayer B, et al. Improvement of liver function in liver cirrhosis patients after autologous mesenchymal stem cell injection: a phase I-II clinical trial. Eur J Gastroenterol Hepatol 2009;21:1199-1205.

37. Amer ME, El-Sayed SZ, El-Kheir WA, et al. Clinical and laboratory evaluation of patients with end-stage liver cell failure injected with bone marrow-derived hepatocyte-like cells. Eur J Gastroenterol Hepatol 2011;23:936-941.

38. Peng L, Xie DY, Lin BL, et al. Autologous bone marrow mesenchymal stem cell transplantation in liver failure patients caused by hepatitis B: short-term and long-term outcomes. Hepatology 2011;54:820-828.

39. El-Ansary M, Abdel-Aziz I, Mogawer S, et al. Phase II trial: undifferentiated versus differentiated autologous mesenchymal stem cells transplantation in Egyptian patients with HCV induced liver cirrhosis. Stem Cell Rev 2012;8:972-981.

40. Shi M, Zhang Z, Xu R, et al. Human mesenchymal stem cell transfusion is safe and improves liver function in acute-on-chronic liver failure patients. Stem Cells Transl Med 2012;1:725-731.

41. Zhang Z, Lin H, Shi M, et al. Human umbilical cord mesenchymal stem cells improve liver function and ascites in decompensated liver cirrhosis patients. J Gastroenterol Hepatol 2012;27 Suppl 2:112-120.

42. Amin MA, Sabry D, Rashed LA, et al. Short-term evaluation of autologous transplantation of bone marrow-derived mesenchymal stem cells in patients with cirrhosis: Egyptian study. Clin Trans- 
plant 2013;27:607-612.

43. Mohamadnejad M, Alimoghaddam K, Bagheri M, et al. Randomized placebo-controlled trial of mesenchymal stem cell transplantation in decompensated cirrhosis. Liver Int 2013;33:1490-1496.

44. Wang L, Li J, Liu H, et al. Pilot study of umbilical cord-derived mesenchymal stem cell transfusion in patients with primary biliary cirrhosis. J Gastroenterol Hepatol 2013;28 Suppl 1:85-92.

45. Jang YO, Kim YJ, Baik SK, et al. Histological improvement following administration of autologous bone marrow-derived mesenchymal stem cells for alcoholic cirrhosis: a pilot study. Liver Int 2014;34:33-41.

46. Salama H, Zekri AR, Medhat E, et al. Peripheral vein infusion of autologous mesenchymal stem cells in Egyptian HCV-positive patients with end-stage liver disease. Stem Cell Res Ther 2014;5:70.

47. Wang L, Han Q, Chen H, et al. Allogeneic bone marrow mesenchymal stem cell transplantation in patients with UDCA-resistant primary biliary cirrhosis. Stem Cells Dev 2014;23:2482-2489.

48. Xu L, Gong Y, Wang B, et al. Randomized trial of autologous bone marrow mesenchymal stem cells transplantation for hepatitis B virus cirrhosis: regulation of Treg/Th17 cells. J Gastroenterol Hepatol 2014;29:1620-1628.

49. Suk KT, Yoon JH, Kim MY, et al. Transplantation with autologous bone marrow-derived mesenchymal stem cells for alcoholic cirrhosis: Phase 2 trial. Hepatology 2016;64:2185-2197.

50. Lanthier N, Lin-Marq N, Rubbia-Brandt L, Clément S, Goossens N, Spahr L. Autologous bone marrow-derived cell transplantation in decompensated alcoholic liver disease: what is the impact on liver histology and gene expression patterns? Stem Cell Res Ther 2017;8:88

51. Lin BL, Chen JF, Qiu WH, et al. Allogeneic bone marrow-derived mesenchymal stromal cells for hepatitis B virus-related acute-onchronic liver failure: a randomized controlled trial. Hepatology 2017;66:209-219.

52. Detry 0, Vandermeulen M, Delbouille MH, et al. Infusion of mesenchymal stromal cells after deceased liver transplantation: a phase I-II, open-label, clinical study. J Hepatol 2017;67:47-55.

53. Meier RP, Müller YD, Morel P, Gonelle-Gispert C, Bühler LH. Transplantation of mesenchymal stem cells for the treatment of liver diseases, is there enough evidence? Stem Cell Res 2013;11:1348-1364.

54. di Bonzo LV, Ferrero I, Cravanzola C, et al. Human mesenchymal stem cells as a two-edged sword in hepatic regenerative medicine: engraftment and hepatocyte differentiation versus profibrogenic potential. Gut 2008;57:223-231.

55. Salama H, Zekri AR, Zern M, et al. Autologous hematopoietic stem cell transplantation in 48 patients with end-stage chronic liver diseases. Cell Transplant 2010;19:1475-1486.

56. Mishra PJ, Mishra PJ, Humeniuk R, et al. Carcinoma-associated fibroblast-like differentiation of human mesenchymal stem cells. Cancer Res 2008;68:4331-4339.

57. Zhou YF, Bosch-Marce M, Okuyama H, et al. Spontaneous transformation of cultured mouse bone marrow-derived stromal cells.
Cancer Res 2006;66:10849-10854.

58. Miura M, Miura Y, Padilla-Nash HM, et al. Accumulated chromosomal instability in murine bone marrow mesenchymal stem cells leads to malignant transformation. Stem Cells 2006;24:1095-1103.

59. Dahl JA, Duggal S, Coulston N, et al. Genetic and epigenetic instability of human bone marrow mesenchymal stem cells expanded in autologous serum or fetal bovine serum. Int J Dev Biol 2008;52:1033-1042.

60. Dhawan A, Puppi J, Hughes RD, Mitry RR. Human hepatocyte transplantation: current experience and future challenges. Nat Rev Gastroenterol Hepatol 2010;7:288-298.

61. Alwahsh SM, Rashidi H, Hay DC. Liver cell therapy: is this the end of the beginning? Cell Mol Life Sci 2018;75:1307-1324.

62. Zeilinger K, Freyer N, Damm G, Seehofer D, Knöspel F. Cell sources for in vitro human liver cell culture models. Exp Biol Med (Maywood) 2016;241:1684-1698.

63. Godoy P, Hewitt NJ, Albrecht U, et al. Recent advances in 2D and $3 \mathrm{D}$ in vitro systems using primary hepatocytes, alternative hepatocyte sources and non-parenchymal liver cells and their use in investigating mechanisms of hepatotoxicity, cell signaling and ADME. Arch Toxicol 2013;87:1315-1530.

64. Li J, Tao R, Wu W, et al. 3D PLGA scaffolds improve differentiation and function of bone marrow mesenchymal stem cell-derived hepatocytes. Stem Cells Dev 2010;19:1427-1436.

65. Liu M, Yang J, Hu W, Zhang S, Wang Y. Superior performance of co-cultured mesenchymal stem cells and hepatocytes in poly(lactic acid-glycolic acid) scaffolds for the treatment of acute liver failure. Biomed Mater 2016;11:015008.

66. Mazza G, Rombouts K, Rennie Hall A, et al. Decellularized human liver as a natural 3D-scaffold for liver bioengineering and transplantation. Sci Rep 2015;5:13079.

67. Deschepper M, Oudina K, David B, et al. Survival and function of mesenchymal stem cells (MSCs) depend on glucose to overcome exposure to long-term, severe and continuous hypoxia. J Cell Mol Med 2011;15:1505-1514.

68. Yu SP, Wei Z, Wei L. Preconditioning strategy in stem cell transplantation therapy. Transl Stroke Res 2013;4:76-88.

69. Haider H, Ashraf M. Preconditioning and stem cell survival. J Cardiovasc Transl Res 2010;3:89-102.

70. Feng Y, Huang W, Meng W, et al. Heat shock improves Sca-1+ stem cell survival and directs ischemic cardiomyocytes toward a prosurvival phenotype via exosomal transfer: a critical role for HSF1/miR-34a/HSP70 pathway. Stem Cells 2014;32:462-472.

71. Qiao PF, Yao L, Zhang XC, Li GD, Wu DQ. Heat shock pretreatment improves stem cell repair following ischemia-reperfusion injury via autophagy. World J Gastroenterol 2015;21:12822-12834.

72. Sandvig I, Gadjanski I, Vlaski-Lafarge M, et al. Strategies to enhance implantation and survival of stem cells after their injection in ischemic neural tissue. Stem Cells Dev 2017;26:554-565.

73. Beegle J, Lakatos K, Kalomoiris S, et al. Hypoxic preconditioning of mesenchymal stromal cells induces metabolic changes, enhances survival, and promotes cell retention in vivo. Stem Cells 
2015;33:1818-1828.

74. Das R, Jahr H, van Osch GJ, Farrell E. The role of hypoxia in bone marrow-derived mesenchymal stem cells: considerations for regenerative medicine approaches. Tissue Eng Part B Rev 2010;16:159-168.

75. Carrière A, Ebrahimian TG, Dehez S, et al. Preconditioning by mitochondrial reactive oxygen species improves the proangiogenic potential of adipose-derived cells-based therapy. Arterioscler Thromb Vasc Biol 2009;29:1093-1099.

76. Alfaifi M, Eom YW, Newsome PN, Baik SK. Mesenchymal stromal cell therapy for liver diseases. J Hepatol 2018;68:1272-1285.

77. Di Rocco G, Gentile A, Antonini A, et al. Enhanced healing of diabetic wounds by topical administration of adipose tissue-derived stromal cells overexpressing stromal-derived factor-1: biodistribution and engraftment analysis by bioluminescent imaging. Stem Cells Int 2010;2011:304562.

78. Mangi AA, Noiseux N, Kong D, et al. Mesenchymal stem cells modified with Akt prevent remodeling and restore performance of infarcted hearts. Nat Med 2003;9:1195-1201.

79. Kutschka I, Kofidis T, Chen IY, et al. Adenoviral human BCL-2 transgene expression attenuates early donor cell death after cardiomyoblast transplantation into ischemic rat hearts. Circulation 2006;114:I174-I180.

80. Liu N, Zhang Y, Fan L, et al. Effects of transplantation with bone marrow-derived mesenchymal stem cells modified by Survivin on experimental stroke in rats. J Transl Med 2011;9:105.

81. Chen S, Chen X, Wu X, et al. Hepatocyte growth factor-modified mesenchymal stem cells improve ischemia/reperfusion-induced acute lung injury in rats. Gene Ther 2017;24:3-11.
82. Hu S, Huang M, Nguyen PK, et al. Novel microRNA prosurvival cocktail for improving engraftment and function of cardiac progenitor cell transplantation. Circulation 2011;124:S27-S34.

83. Xu J, Huang $\mathrm{Z}$, Lin L, et al. miR-210 over-expression enhances mesenchymal stem cell survival in an oxidative stress environment through antioxidation and c-Met pathway activation. Sci China Life Sci 2014;57:989-997.

84. Camussi G, Deregibus MC, Cantaluppi V. Role of stem-cell-derived microvesicles in the paracrine action of stem cells. Biochem Soc Trans 2013;41:283-287.

85. Koppers-Lalic D, Hogenboom MM, Middeldorp JM, Pegtel DM. Virus-modified exosomes for targeted RNA delivery; a new approach in nanomedicine. Adv Drug Deliv Rev 2013;65:348-356.

86. Kooijmans SAA, Schiffelers RM, Zarovni N, Vago R. Modulation of tissue tropism and biological activity of exosomes and other extracellular vesicles: new nanotools for cancer treatment. Pharmacol Res 2016;111:487-500.

87. Li T, Yan Y, Wang B, Qian H, et al. Exosomes derived from human umbilical cord mesenchymal stem cells alleviate liver fibrosis Stem Cells Dev 2013;22:845-854.

88. Nong K, Wang W, Niu X, et al. Hepatoprotective effect of exosomes from human-induced pluripotent stem cell-derived mesenchymal stromal cells against hepatic ischemia-reperfusion injury in rats. Cytotherapy 2016;18:1548-1559.

89. Du Y, Li D, Han C, et al. Exosomes from human-induced pluripotent stem cell-derived mesenchymal stromal cells (hiPSC-MSCs) protect liver against hepatic ischemia/reperfusion injury via activating sphingosine kinase and sphingosine-1-phosphate signaling pathway. Cell Physiol Biochem 2017;43:611-625. 